Hurşit Önsiper · Sinan Sertöz

\title{
Generalized Shioda-Inose structures on K3 surfaces
}

Received: 9 April 1998 / Revised version: 17 July 1998

\begin{abstract}
In this note, we study the action of finite groups of symplectic automorphisms on K3 surfaces which yield quotients birational to generalized Kummer surfaces. For each possible group, we determine the Picard number of the K3 surface admitting such an action and for singular K3 surfaces we show the uniqueness of the associated abelian surface.
\end{abstract}

This work concerns algebraic K3 surfaces admitting generalized ShiodaInose structures (Definition 1 below). To generalize the classical ShiodaInose structure ([S-I], $[\mathrm{M}]$ ), one needs to determine finite groups with suitable actions both on K3 surfaces and on abelian surfaces. To this end, finite groups with symplectic actions on $\mathrm{K} 3$ surfaces were completely determined in ([Mu2]) and ([X]) and in the latter article the configurations of singularities on the quotients were also listed. On the complementary side, Katsura's article $([\mathrm{K}])$ contains the classification of all finite groups acting on abelian surfaces so as to yield generalized Kummer surfaces (cf. [B] for related lattice theoretic discussion).

In this paper, using the results of $([\mathrm{K}, \mathrm{X}])$ we show that a $\mathrm{K} 3$ surface $X$ admitting a Shioda-Inose structure with $G \neq \mathbf{Z}_{2}$ has $\rho(X) \geq 19$ in general and $\rho(X)=20$ if $G$ is noncyclic. We also show that on a singular $\mathrm{K} 3$ surface $X$, all Shioda-Inose structures are induced by a unique abelian surface.

Throughout the paper we will consider only algebraic K3 surfaces over C.

Our notation will be as follows:

$A$ (resp. $X$ ) denotes an abelian (resp. an algebraic K3) surface.

$A_{G}$ is the Kummer surface constructed from $A / G$ for a suitable finite group $G$.

H. Önsiper: Department of Mathematics, Middle East Technical University, 06531 Ankara, Turkey. e-mail: hursit@ rorqual.cc.metu.edu.tr

S. Sertöz: Department of Mathematics, Bilkent University, Ankara, Turkey.

e-mail: sertoz@fen.bilkent.edu.tr

Mathematics Subject Classification (1991): 14J28 
$K$ ? denotes the canonical class of ?.

$T_{?}=$ the transcendental lattice of ?.

$\rho(?)$ is the Picard number of ?.

We use the standard notation $A_{k}, D_{k}, E_{k}$ to denote the rational singularities on surfaces.

$|G|$ denotes the order of the group $G$.

We begin with giving a precise definition of Shioda-Inose structures on K3 surfaces. For this, we first recall that a generalized Kummer surface $A_{G}$ is a $\mathrm{K} 3$ surface which is the minimal resolution of the quotient $A / G$ of an abelian surface $A$ by some finite group $G$ ([K], Definition 2.1).

Definition 1. A K3 surface $X$ admits a Shioda-Inose structure with group $G$ if $G$ acts on $X$ symplectically and the quotient $X / G$ is birational to a generalized Kummer surface $A_{G}$.

We note that generalized Kummer surfaces (in characteristic 0 ) arise only if $G$ is isomorphic to one of the following groups ([K], Corollary 3.17):

$\mathbf{Z}_{k}, k=2,3,4,6$,

binary dihedral groups $Q_{8}, Q_{12}$ and

binary tetrahedral group $T_{24}$.

All of these possibilities occur ([K], Examples).

Comparing this list with the list of finite groups acting symplectically on K3 surfaces ([X], Table 2), we see that all such $G$ appear as a group of symplectic automorphisms of some K3 surface. Hence the question of the existence of K3 surfaces admitting Shioda-Inose structures with group $G$ makes sense for each of these groups $G$.

In the classical case of $G=\mathbf{Z}_{2}$, Morrison obtained the following lattice theoretic characterization of K3 surfaces admitting Shioda-Inose structure ([M], Corollary 6.4).

Theorem [M]. An algebraic K3 surface X admits a Shioda-Inose structure if and only if $X$ satisfies one of the following conditions:

(i) $\rho(X)=19$ or 20 ,

(ii) $\rho(X)=18$ and $T_{X}=U \oplus T^{\prime}$,

(iii) $\rho(X)=17$ and $T_{X}=U^{2} \oplus T^{\prime}$

where $U$ is the standard hyperbolic lattice.

To contrast this case with the general situation, we include the following elementary observation.

Lemma 2. Given an abelian surface $A$, there exists a K3 surface $X$ with $\rho(X)=16+\rho(A)$ admitting a classical Shioda-Inose structure induced by $A$. 
Proof. Given $A$, we have $T_{A} \hookrightarrow U^{3}$ with signature $\left(T_{A}\right)=(2,4-\rho(A))$. Therefore, taking $\rho=16+\rho(A)$, by the surjectivity of the period map for K3 surfaces (cf. [M], Corollary 1.9 (ii)) we have a K3 surface $X$ with $\rho(X)=\rho$ and $T_{X}$ is isometric to $T_{A}$. Applying ([M], Theorem 6.3) the conclusion follows.

We will see that for generalized Shioda-Inose structures one has $\rho(X) \geq$ 19. This bound on the Picard number follows from the configuration of the exceptional curves on $A_{G}$ for which we will need the following result on the singularities of the quotient $A / G$ for noncyclic $G$.

Proposition 3. If $G$ is a non-cyclic group acting on an abelian surface A to yield a generalized Kummer surface, then the singularities of $A / G$ are given as follows:

$$
\begin{aligned}
& 3 A_{1}+4 D_{4} \text { for } G=Q_{8}, \\
& A_{1}+2 A_{2}+3 A_{3}+D_{5} \text { for } G=Q_{12} \text { and } \\
& 4 A_{2}+2 A_{3}+A_{5} \text { or } A_{1}+4 A_{2}+D_{4}+E_{6} \text { for } G=T_{24} .
\end{aligned}
$$

Proof. As is the case with analysis of this type, the proof is combinatorial in essence and is quite standard (cf. [B], [K], [X]). We know that as we have only quotient singularities, the possible types of singularities are:

$A_{k}, k=1,2,3,5$ corresponding to stabilizer groups of type $\mathbf{Z}_{k}, k=$ $2,3,4,6$ respectively and $D_{4}$ (resp. $D_{5}$, resp. $E_{6}$ ) corresponding to $Q_{8}$ (resp. $Q_{12}$, resp. $T_{24}$ ). We index these types in this order with $i=1, . ., 7$ and we let $n_{i}$ be the number of singular points of type $i$ on $A / G$.

Comparing the topological Euler characteristic of $A-\{$ fixed points of $G$ \} to that of $A_{G}-\{$ exceptional curves $\}$, we obtain

$$
0=\chi_{\text {top }}(A)=|G|\left(24-\sum \chi_{i} n_{i}\right)+n,
$$

where $\chi_{i}$ is the topological Euler characteristic of the configuration corresponding to the singularity of type $i$ and $n$ is the total number of fixed points of $G$ on $A$. Clearly we have $n=\sum m_{i} n_{i}$ where $m_{i}$ is the index in $G$ of the stabilizer group corresponding to $i$. Furthermore, as the lattice generated by (-2)-curves on $A_{G}$ has rank $\leq 19$, in all cases we have

$$
n_{1}+2 n_{2}+3 n_{3}+5 n_{4}+4 n_{5}+5 n_{6}+6 n_{7} \leq 19 .
$$

Using these restrictions together with the subgroup structure of each $G$, the result follows.

Corollary 4. If $X$ admits a Shioda-Inose structure with $G \neq \mathbf{Z}_{2}$, then $\rho(X) \geq 19$ and $\rho(X)=20$ if $G$ is noncyclic. 
Proof. By ([I2], Corollary 1.2), we know that the Picard number of $X$ is equal to the Picard number of the associated generalized Kummer surface $A_{G}$. Therefore, if $G=\mathbf{Z}_{k}$ for $k=3,4,6$, it follows from ([K], p. 17) that $\rho(X) \geq 19$. In case $G$ is noncyclic, we apply Proposition 3 to see that $\rho(X)=20$.

Next, we consider the variation of Shioda-Inose structures with respect to the isogenies of abelian surfaces.

Given a K3-surface $X$ which admits a Shioda-Inose structure with group $G$ and associated abelian surface $A$, we denote by $\pi_{A}$ (resp. $\pi_{X}$ ) the rational covering map $A \rightarrow A_{G}$ (resp. $X \rightarrow A_{G}$ ) into the corresponding generalized Kummer surface $A_{G}$.

The following results follow by exactly the same proofs as in the case of classical Shioda-Inose structures (cf. [S-I], [I2], [M]):

(1) $\pi_{A}^{*}\left(K_{A_{G}}\right)=K_{A}$ and $\pi_{X}^{*}\left(K_{A_{G}}\right)=K_{X}$,

(2) $\pi_{A}^{*}: T_{A_{G}} \rightarrow T_{A}$ (resp. $\pi_{X}^{*}$ ) gives an isomorphism of lattices $T_{A_{G}} \cong$ $T_{A}(n)\left(\right.$ resp. $\left.T_{A_{G}} \cong T_{X}(n)\right)$ where $n=|G|$,

(3) $T_{A}$ and $T_{X}$ are isometric.

Using these elementary observations we prove

Lemma 5. If $X$ is a singular $K 3$ surface, then each and every Shioda-Inose structure on $X$ is induced only by $A$.

Proof. We let $p_{A}, p_{A_{G}}, p_{X}$ denote the period maps of $A, A_{G}, X$ respectively.

From (1) above, it follows that the isometry $\phi: T_{X} \rightarrow T_{A}$ satisfies $p_{A} \circ \phi=c p_{X}$ for some $c \in \mathbf{C}$.

If we have another abelian surface $A^{\prime}$ inducing some Shioda-Inose structure on $X$, with corresponding isometry $\psi: T_{X} \rightarrow T_{A^{\prime}}$ satisfying $p_{A^{\prime}} \circ \psi=c^{\prime} p_{X}$ for some $c^{\prime} \in \mathbf{C}$, then we get an isometry $\phi \circ \psi^{-1}: T_{A^{\prime}} \rightarrow T_{A}$. As $\rho(A)=$ $4, \phi \circ \psi^{-1}$ extends to an isometry $\alpha: H^{2}\left(A^{\prime}, \mathbf{Z}\right) \rightarrow H^{2}(A, \mathbf{Z})$ ([S-M], Theorem 1 in Appendix) to give $p_{A} \circ \alpha=p_{A} \circ\left(\phi \circ \psi^{-1}\right)=c c^{-1} p_{A^{\prime}}$, and it follows that $A^{\prime} \cong A$ or the dual $\hat{A}$ of $A$ ([S], Theorem 1). This completes the proof because $X$ admits a classical Shioda-Inose structure for which the associated abelian surface $A^{\prime}$ is self-dual (Theorem [S-I]).

Remark. If $A_{1}$ and $A_{2}$ are two abelian surfaces which are isogeneous, then we have $T_{A_{1}} \otimes \mathbf{Q} \cong T_{A_{2}} \otimes \mathbf{Q}$. Therefore two K3 surfaces $X_{1}, X_{2}$ are isogeneous in the sense of ([Mu1], Definition 1.8) if they admit Shioda-Inose structures (not necessarily with the same group) induced from $A_{1}, A_{2}$ respectively ([Mu1], Remark 1.11). In case $X_{1}, X_{2}$ are singular K3 surfaces, the stronger form of isogeny follows from Lemma 5 using ([I1]); that is, we have rational maps $X_{1} \rightarrow X_{2}, X_{2} \rightarrow X_{1}$ of finite degree. 


\section{References}

[B] Bertin, J.: Resaux de Kummer et surfaces K3. Invent. Math. 93, 267-284 (1988)

[I1] Inose, H.: On defining equations of singular K3 surfaces and a notion of isogeny. In: Proc. Int. Symp. Alg. Geo., Kyoto 1977, Kinokuniya Books 1978, pp. 495-502

[12] Inose, H.: On certain Kummer surfaces which can be realized as nonsingular quartic surfaces in $\mathbf{P}^{\mathbf{3}}$. J. Fac. Sci. Univ. Tokyo Sect. IA Math. 23 No 3, 545-560 (1976)

[K] Katsura, T.: Generalized Kummer surfaces and their unirationality in characteristic p. J. Fac. Sci. Univ. Tokyo, Sect. IA, Math. 34, 1-41 (1987)

[M] Morrison, D.: On K3 surfaces with large Picard number. Inv. Math. 75, 105-121 (1984)

[Mu1] Mukai, S.: On the moduli spaces of bundles on K3 surfaces I. In: Vector Bundles in Algebraic Geometry Tata Institute of Fundamental Research Studies 11, Oxford: Oxford Uni. Press 1987, pp. 341-413

[Mu2] Mukai, S.: Finite groups of automorphisms of K3 surfaces and the Mathieu group. Inv. Math. 94, 183-221 (1988)

[S] Shioda, T.: The period map of abelian surfaces. J. Fac. Sci. Uni. Tokyo, 25, 47-59 (1978)

[S-I] Shioda, T., Inose, H.: On singular K3 surfaces. In: Complex Analysis and Algebraic Geometry, Iwanami Shoten, 1977, pp. 119-136

[S-M] Shioda, T., Mitani, N.: Singular abelian surfaces and binary quadratic forms. In: Classification of Algebraic Varieties and Compact Complex Manifolds, LNM No. 412, 259-287 (1974)

[X] Xiao, G.: Galois covers between K3 surfaces. Ann. Inst. Fourier, Grenoble 46, 73-88 (1996) 